\title{
A SUFFICIENT CONDITION FOR NEVANLINNA PARAMETRIZATION AND AN EXTENSION OF HEINS THEOREM
}

\author{
SECHIKO TAKAHASHI
}

\begin{abstract}
An extended interpolation problem on a Riemann surface is formulated in terms of local rings and ideals. A sufficient condition for Nevanlinna parametrization is obtained. By means of this, Heins theorem on PickNevanlinna interpolation in doubly connected domains is generalized to extended interpolation.
\end{abstract}

\section{$\S 1$. Introduction}

In order to consider extended interpolation problems on Riemann surfaces and to make simple the expressions concerning transformations, we wish at first to give a formulation of extended interpolation problems in terms of local rings and ideals.

Let $X$ be a Riemann surface, i.e. a connected 1-dimensional complex manifold. For each $x \in X, \mathcal{O}_{x}$ denotes the ring of germs of holomorphic functions at $x$. Consider for each $x \in X$ a nonzero ideal $\mathcal{I}_{x}$ of $\mathcal{O}_{x}$ and an element $\mathfrak{c}_{x}$ of the quotient ring $\mathcal{O}_{x} / \mathcal{I}_{x}$. The collection $(\mathcal{I}, \mathfrak{c})$, where $\mathcal{I}=\left(\mathcal{I}_{x}\right)_{x \in X}$ and $\mathfrak{c}=\left(\mathfrak{c}_{x}\right)_{x \in X}$, will be called extended interpolation problem on $X$. Let $\mathcal{B}$ denote the set of all holomorphic functions $f$ on $X$ such that $|f| \leq 1$ on $X$. Our problem is to find a function $f \in \mathcal{B}$ which satisfies the condition

$$
f_{x}+\mathcal{I}_{x}=\mathfrak{c}_{x} \quad(\forall x \in X),
$$

where $f_{x}$ is the germ at $x$ represented by $f$ and $f_{x}+\mathcal{I}_{x}$ is the coset of $f_{x}$ modulo $\mathcal{I}_{x}$. Such a function will be called solution in $\mathcal{B}$ of the problem $(\mathcal{I}, \mathfrak{c})$. Let

$$
\mathcal{E}=\{f \in \mathcal{B}: f \text { satisfies }(1.1)\}
$$

denote the set of solutions in $\mathcal{B}$ of the problem $(\mathcal{I}, \mathfrak{c})$. Some remarks will be added in $\S 2$.

Received July 14, 1997.

Revised January 20, 1998. 
In $\S 4$, we suppose that $X$ is biholomorphically equivalent to the open unit disc $D=\{z \in \mathbb{C}:|z|<1\}$ and that $\mathcal{E}$ has at least two elements. We showed in [14] that, under these hypotheses, there exists a Nevanlinna parametrization $\pi$ of $\mathcal{E}$, which is by definition a bijection $\pi: \mathcal{B} \longrightarrow \mathcal{E}$ such that we have for any $g \in \mathcal{B}$

$$
\pi(g)=\frac{P g+Q}{R g+S} \quad \text { with } \quad R g+S \not \equiv 0 \text { (not equal to the constant zero) },
$$

where $P, Q, R$, and $S$ are holomorphic functions on $X$, independent of $g$. The quadruple $(P, Q, R, S)$ is said to represent $\pi$. In $\S 4$, under those hypotheses, we give a sufficient condition for a quadruple of the form $(P, Q, R, 1)$, where $P, Q$, and $R$ are holomorphic on $X$, to represent a Nevanlinna parametrization of $\mathcal{E}$.

In $\S 5$, supposing $X$ is as above, one considers an analytic automorphism $T$ of $X$ and a Möbius transformation $U$ of the closed unit disc $\bar{D}=\{z \in$ $\mathbb{C}:|z| \leq 1\}$. In the case of classical Pick-Nevanlinna interpolation, where $\mathcal{I}_{x}$ is equal to the unique maximal ideal $\mathfrak{m}_{\mathfrak{x}}$ of $\mathcal{O}_{x}$ or to the whole ring $\mathcal{O}_{x}$, Heins showed in [5] that, under the consistency conditions, imposed on $\mathcal{I}$ and $\mathfrak{c}$, and the assumption $\mathcal{E} \neq \emptyset$, there exists a solution $f \in \mathcal{E}$ such that $f \circ T=U \circ f$. We generalize Heins theorem to our extended interpolation, based essentially on Heins' method. But, we use the result obtained in $\S 4$, which would make the proof transparent. The result of Heins on the classical interpolation in annuli in [5] is also generalized.

\section{§. Preliminaries}

As in $\S 1$, consider an extended interpolation problem $(\mathcal{I}, \mathfrak{c})$ on a Riemann surface $X$. Let $\mathcal{B}$ and $\mathcal{E}$ be as in $\S 1$ and consider the set $\sigma=\{x \in$ $\left.X: \mathcal{I}_{x} \neq \mathcal{O}_{x}\right\}$.

We would like to give some remarks on the above formulation. If $\mathcal{I}_{x}=$ $\mathcal{O}_{x}$ then $\mathcal{O}_{x} / \mathcal{I}_{x}$ and hence $\mathfrak{c}_{x}$ reduce to zeros, so that there is no requirements at $x$. Suppose $x \in \sigma$. Then, as $\{0\} \neq \mathcal{I}_{x} \neq \mathcal{O}_{x}$, there is a unique positive integer $n_{x}$ such that $\mathcal{I}_{x}=\mathfrak{m}_{\mathfrak{x}}^{\mathfrak{n}_{\mathfrak{x}}}$, where $\mathfrak{m}_{\mathfrak{x}}$ is the maximal ideal of the local ring $\mathcal{O}_{x}$. Associating to $x$ a local coordinate $z$ such that $z(x)=0$, we obtain

$$
\mathfrak{c}_{x}=\left(c_{0}+c_{1} z+\cdots+c_{n_{x}-1} z^{n_{x}-1}\right)_{x}+\mathcal{I}_{x},
$$

where the $n_{x}$ and the constants $c_{0}, c_{1}, \cdots, c_{n_{x}-1}$ are uniquely determined by $\mathfrak{c}_{x}$ and $z$. To give an extended interpolation problem $(\mathcal{I}, \mathfrak{c})$ is thus to give 
for each $x \in \sigma$ a positive integer $n_{x}$, a local coordinate $z$ at $x$, and first $n_{x}$ Taylor coefficients $c_{0}, c_{1}, \cdots, c_{n_{x}-1}$ with respect to $z$.

For an arbitrary $\mathcal{I}=\left(\mathcal{I}_{x}\right)_{x \in X}$ and for any $f \in \mathcal{B}$, setting $\mathfrak{c}_{x}=f_{x}+\mathcal{I}_{x}$ for each $x \in X$, we have an extended interpolation problem $(\mathcal{I}, \mathfrak{c})$, which admits at least $f$ as a solution in $\mathcal{B}$. In this case, $\sigma$ may be arbitrary.

However, the case where $\sigma$ is nonempty and has no limit points in $X$, i.e. $\sigma$ is a discrete closed set of $X$, is of prime importance. For example, when $\mathcal{E}$ has at least two elements, $\sigma$ has no limit points in $X$. In such a case, $\mathcal{I}=\bigcup_{x \in X} \mathcal{I}_{x}$ may be regarded as a coherent analytic subsheaf of the structure sheaf $\mathcal{O}=\bigcup_{x \in X} \mathcal{O}_{x}$ of $X, \mathfrak{c}$ as an element of the cohomology group $H^{0}(X, \mathcal{O} / \mathcal{I})$, and $f \in \mathcal{E}$ as an element of $H^{0}(X, \mathcal{O}) \cap \mathcal{B}$ which is mapped to $\mathfrak{c}$ by the canonical homomorphism $H^{0}(X, \mathcal{O}) \longrightarrow H^{0}(X, \mathcal{O} / \mathcal{I})$.

Let $X, Y$ be Riemann surfaces and let $\varphi: X \longrightarrow Y$ be a holomorphic mapping. For each $x \in X$, setting $y=\varphi(x)$, we have the canonical ring homomorphism $\varphi_{x}^{*}: \mathcal{O}_{y} \longrightarrow \mathcal{O}_{x}$ defined by

$$
\varphi_{x}^{*}\left(g_{y}\right)=(g \circ \varphi)_{x},
$$

where $g$ is a holomorphic function at $y$ on $Y$. Moreover, when an ideal $\mathcal{I}_{x}$ of $\mathcal{O}_{x}$ and an ideal $\mathcal{I}_{y}$ of $\mathcal{O}_{y}$ are given in such a way that $\varphi_{x}^{*}\left(\mathcal{I}_{y}\right) \subset \mathcal{I}_{x}$, we have a canonical ring homomorphism $\mathcal{O}_{y} / \mathcal{I}_{y} \longrightarrow \mathcal{O}_{x} / \mathcal{I}_{x}$, which will be denoted by the same symbol $\varphi_{x}^{*}$.

In $\S 5$, we shall be concerned with a Möbius transformation of functions, so that we wish here to introduce a notation and to make some remarks. Consider a linear transformation $U$ of the Riemann sphere:

$$
U(w)=\frac{p w+q}{r w+s} \quad(p, q, r, s \in \mathbb{C} ; p s-q r \neq 0) .
$$

Let $x \in X$ and let $\mathcal{I}_{x}$ be an ideal of $\mathcal{O}_{x}$ such that $\{0\} \neq \mathcal{I}_{x} \neq \mathcal{O}_{x}$. For an element $\mathfrak{c}_{x} \in \mathcal{O}_{x} / \mathcal{I}_{x}$, whenever $r \mathfrak{c}_{x}+s$ is a unit of the ring $\mathcal{O}_{x} / \mathcal{I}_{x}$, we can define $U_{x}\left(\mathfrak{c}_{x}\right) \in \mathcal{O}_{x} / \mathcal{I}_{x}$ by

$$
U_{x}\left(\mathfrak{c}_{x}\right)=\frac{p \mathfrak{c}_{x}+q}{r \mathfrak{c}_{x}+s},
$$

where $p, q, r$, and $s$ are regarded as elements of $\mathcal{O}_{x} / \mathcal{I}_{x}$ represented by constant functions. If $f$ is a holomorphic function in a neighborhood of $x$ and if $\mathfrak{c}_{x}=f_{x}+\mathcal{I}_{x}$, then one sees immediately that $r \mathfrak{c}_{x}+s$ is a unit if and only if $r f(x)+s \neq 0$ and that, if so, we have

$$
U_{x}\left(\mathfrak{c}_{x}\right)=\left(\frac{p f+q}{r f+s}\right)_{x}+\mathcal{I}_{x} .
$$


We would like to point out that, if $U$ is a Möbius transformation, i.e. $U(\bar{D})=\bar{D}$, and if we have $\mathfrak{c}_{x}=f_{x}+\mathcal{I}_{x}$ for some holomorphic function $f$ in a neighborhood of $x$ with $|f(x)| \leq 1$, then we have $|r|<|s|$ and hence $r \mathfrak{c}_{x}+s$ is a unit, so that $U_{x}\left(\mathfrak{c}_{x}\right)$ can be defined.

For the holomorphic mapping $\varphi: X \longrightarrow Y$, let $y=\varphi(x)$ and suppose $\{0\} \neq \mathcal{I}_{y} \neq \mathcal{O}_{y}$ and $\varphi_{x}^{*}\left(\mathcal{I}_{y}\right) \subset \mathcal{I}_{x}$. Let $\mathfrak{c}_{y} \in \mathcal{O}_{y} / \mathcal{I}_{y}$. If $r \mathfrak{c}_{y}+s$ is a unit of $\mathcal{O}_{y} / \mathcal{I}_{y}$, then one sees at once that $r \varphi_{x}^{*}\left(\mathfrak{c}_{y}\right)+s$ is also a unit of $\mathcal{O}_{x} / \mathcal{I}_{x}$ and that we have

$$
U_{x}\left(\varphi_{x}^{*}\left(\mathfrak{c}_{y}\right)\right)=\frac{p \varphi_{x}^{*}\left(\mathfrak{c}_{y}\right)+q}{r \varphi_{x}^{*}\left(\mathfrak{c}_{y}\right)+s}=\varphi_{x}^{*}\left(\frac{p \mathfrak{c}_{y}+q}{r \mathfrak{c}_{y}+s}\right)=\varphi_{x}^{*}\left(U_{y}\left(\mathfrak{c}_{y}\right)\right)
$$

\section{$\S 3$. Nevanlinna parametrizations}

Based on Nevanlinna's method([9]), we defined and studied Nevanlinna parametrizations in [14]. In this section, we wish to restate, in terms of the formulation introduced in the previous sections, the definition and some results, obtained in [14] and needed below.

Let $X$ be a simply connected Riemann surface of hyperbolic type; that is, $X$ is biholomorphically equivalent to the open unit disc $D$. Let $(\mathcal{I}, \mathfrak{c})$, where $\mathcal{I}=\left(\mathcal{I}_{x}\right)_{x \in X}$ and $\mathfrak{c}=\left(\mathfrak{c}_{x}\right)_{x \in X}$, be an extended interpolation problem on $X$. Let $\mathcal{B}$ denote the set of all holomorphic functions $f$ on $X$ with $|f| \leq 1$ on $X$ and let $\mathcal{E}$ denote the set of all solutions in $\mathcal{B}$ of the problem $(\mathcal{I}, \mathfrak{c})$.

A Nevanlinna parametrization $\pi$ is by definition a bijection $\pi: \mathcal{B} \longrightarrow \mathcal{E}$ represented by a quadruple $(P, Q, R, S)$ of four holomorphic functions on $X$ in such a way that

$$
\pi(g)=\frac{P g+Q}{R g+S}, \quad R g+S \not \equiv 0 \quad(\forall g \in \mathcal{B}) .
$$

It is well known ([9] and [14]) that $\mathcal{E}$ admits a Nevanlinna parametrization if and only if $\mathcal{E}$ has at least two elements. From now on in $\S 3$ as well as in $\S 4$, we assume that $\mathcal{E}$ has at least two elements and that the set $\sigma=\left\{x \in X: \mathcal{I}_{x} \neq \mathcal{O}_{x}\right\}$ is nonempty. Then $\sigma$ has no limit points in $X$ and there exists a Blaschke product $B$ on $X$ associated to $\mathcal{I}=\left(\mathcal{I}_{x}\right)_{x \in X}$. In fact, for two distinct elements $f_{0}$ and $f_{1}$ of $\mathcal{E}$, we see that the function $h=f_{0}-f_{1}(\not \equiv 0)$ is bounded on $X$, and that each $x \in \sigma$ is a zero of $h$ of order at least $n_{x}$, where $n_{x}$ is the positive integer such that $\mathcal{I}_{x}=\mathfrak{m}_{\mathfrak{x}}^{\mathfrak{n}_{\mathfrak{x}}}$. Take 
a biholomorphic mapping $\theta: X \longrightarrow D$ and write

$$
\begin{aligned}
& B(x)=\prod_{x_{i} \in \sigma}\left(\lambda_{i} \frac{\theta(x)-z_{i}}{1-\overline{z_{i}} \theta(x)}\right)^{n_{i}}, \\
& \lambda_{i}=\left\{\begin{array}{ll}
1 & \left(\text { if } z_{i}=0\right) \\
-\left|z_{i}\right| / z_{i} & \left(\text { if } z_{i} \neq 0\right)
\end{array},\right.
\end{aligned}
$$

where $z_{i}=\theta\left(x_{i}\right), n_{i}=n_{x_{i}}$. It is not hard to see that $B$ is a required Blaschke product on $X$.

If $(P, Q, R, S)$ represents a Nevanlinna parametrization $\pi$ of $\mathcal{E}$, then these functions have the following properties (Proposition 3 and Corollary 2 of [14]. See also Notes in $\S 5$ of [14]):

(a) $S \not \equiv 0$.

(b) $|P / S|<1,|Q / S|<1,|R / S|<1$ on $X$.

(c) $Q / S \in \mathcal{E}$.

(d) We may write

$$
\frac{P S-Q R}{S^{2}}=U \cdot B,
$$

where $U$ is a holomorphic function on $X$ such that $0<|U| \leq 1$ on $X$ and $B$ is a Blaschke product on $X$ associated to $\mathcal{I}$.

By virtue of these properties, we may assume $S \equiv 1$. If two quadruples $(P, Q, R, S)$ and $(\widehat{P}, \widehat{Q}, \widehat{R}, \widehat{S})$ represent the same $\pi$, then there exists a meromorphic function $M$ on $X$ such that $(\widehat{P}, \widehat{Q}, \widehat{R}, \widehat{S})=M(P, Q, R, S)$. Hence, each Nevanlinna parametrization of $\mathcal{E}$ is represented by one and only one quadruple of the form $(P, Q, R, 1)$.

Let $\mathcal{P}$ denote the set of all Nevanlinna parametrizations of $\mathcal{E}$ and let $G$ denote the group of all Möbius transformations

$$
\tau(w)=\lambda \frac{w+a}{1+\bar{a} w} \quad(\lambda, a \in \mathbb{C} ;|\lambda|=1,|a|<1)
$$

regarded as analytic automorphisms of the closed unit disc $\bar{D}=D \bigcup \partial D$. The group $G$ operates on $\mathcal{P}$ in the following way:

$$
\left(\tau^{*}(\pi)\right)(g)=\pi(\tau \circ g) \quad(\tau \in G, \pi \in \mathcal{P}, g \in \mathcal{B}) .
$$

We obtained (Theorem 1 of [14])

TheOREM 1. Let $\pi, \pi_{0} \in \mathcal{P}$. Then there exists one and only one $\tau \in G$ such that $\pi=\tau^{*}\left(\pi_{0}\right)$. 
For each $x \in X$, let

$$
W(x)=\{f(x): f \in \mathcal{E}\}
$$

denote the set of values at $x$ taken by all solutions $f \in \mathcal{E}$. We have $W(x) \subset$ $D$. To each $\pi \in \mathcal{P}$ and to each $x \in X$, we associate the mapping $\pi_{x}$ : $\bar{D} \longrightarrow W(x)$ defined by

$$
\pi_{x}(\zeta)=\pi(\zeta)(x) \quad(\zeta \in \bar{D})
$$

where $\zeta$ is regarded as a constant function. If $\pi$ is represented by $(P, Q, R, S)$, we have

$$
\pi_{x}(\zeta)=\frac{P(x) \zeta+Q(x)}{R(x) \zeta+S(x)} \quad(\zeta \in \bar{D})
$$

If $x \in X \backslash \sigma$, then $\pi_{x}$ is bijective and $W(x)$ is a nondegenerate closed disc in $D$.

We showed (Lemma 1 of [14])

Theorem 2. Let $x \in X \backslash \sigma, w \in \partial W(x)$. Then there is a unique $f \in \mathcal{E}$ such that $f(x)=w$.

About the extremal solutions, we established (Corollary 3 of [14])

Theorem 3. Assume that $\mathcal{E}$ has at least two elements. Let $\pi \in \mathcal{P}$ and $f \in \mathcal{E}$. The following three conditions are equivalent:

(a) $f(x) \in \partial W(x)$ for some $x \in X \backslash \sigma$.

(b) $f(x) \in \partial W(x)$ for all $x \in X \backslash \sigma$.

(c) There exists a $\zeta \in \partial D$ such that $f=\pi(\zeta)$.

If one of these conditions is satisfied, $\zeta$ in (c) is uniquely determined by $\pi$ and $f$.

\section{§4. A sufficient condition for parametrization}

Let $(\mathcal{I}, \mathfrak{c})$ be an extended interpolation problem on a simply connected Riemann surface $X$ of hyperbolic type. Let $\mathcal{B}, \mathcal{E}$, and $\sigma$ be as in $\S 3$ and assume $\mathcal{E}$ has at least two elements. Consider three holomorphic functions $P, Q$, and $R$ on $X$. In this section, we want to give a sufficient condition 
for the quadruple $(P, Q, R, 1)$ to represent a Nevanlinna parametrization of $\mathcal{E}$. To this end, consider for each $x \in X$ two sets

$$
\delta(x)=\left\{\frac{P(x) \zeta+Q(x)}{R(x) \zeta+1}: \zeta \in \bar{D}, R(x) \zeta+1 \neq 0\right\}
$$

and

$$
W(x)=\{f(x): f \in \mathcal{E}\} .
$$

We recall here well-known properties concerning linear transformations :

Proposition. Let

$$
\tau(w)=\frac{p w+q}{r w+s} \quad(p, q, r, s \in \mathbb{C} ; p s-q r \neq 0)
$$

be a linear transformation of the Riemann sphere. If $\tau(\bar{D}) \subset D$ then we have $|p|<|s|,|q|<|s|$, and $|r|<|s|$

We begin with the following

Lemma. Suppose $\delta(x) \subset W(x)$ for all $x \in X$.

(a) Then we have

$$
\frac{P g+Q}{R g+1} \in \mathcal{E} \quad(\forall g \in \mathcal{B}) .
$$

(b) Moreover, if there is a point $x_{0} \in X \backslash \sigma$ such that $\delta\left(x_{0}\right)=W\left(x_{0}\right)$, then we have $\delta(x)=W(x)$ for all $x \in X$.

First of all, we remark something about $P-Q R$. If $P(x)-Q(x) R(x) \neq 0$ for a point $x \in X \backslash \sigma$, then, by the assumption $\delta(x) \subset W(x) \subset D$ and by the above proposition, we see easily $|R(x)|<1$. Thus, if $P-Q R \not \equiv 0$, then we have $|R|<1$ on $X$ and the condition $R(x) \zeta+1 \neq 0$ in the definition of $\delta(x)$ can be omitted. If $P-Q R \equiv 0$, then $\delta(x)$ degenerates to a sole point $Q(x)$ for any $x \in X$ and, in (a) of the lemma, we may set $(P g+Q) /(R g+1)=Q$ for any $g \in \mathcal{B}$, even in the case $R g+1 \equiv 0$.

Proof. To see (a), take a Nevanlinna parametrization $\widehat{\pi}$ of $\mathcal{E}$ represented by a quadruple $(\widehat{P}, \widehat{Q}, \widehat{R}, 1)$ and let $g \in \mathcal{B}$. In any case, $f=(P g+Q) /(R g+$ 1 ) is holomorphic on $X$ and we have $f(x) \in \delta(x) \subset W(x)$ for any $x \in X$. 
Since, for each $x \in X \backslash \sigma$, the mapping $\widehat{\pi}_{x}: \bar{D} \longrightarrow W(x)$, defined in $\S 3$, is bijective, the function

$$
\widehat{g}(x)=\widehat{\pi}_{x}^{-1}(f(x))=\frac{f(x)-\widehat{Q}(x)}{-\widehat{R}(x) f(x)+\widehat{P}(x)}
$$

is well-defined and holomorphic on $X \backslash \sigma$ and satisfies $|\widehat{g}| \leq 1$ there. Hence $\widehat{g}$ may be regarded as a function in $\mathcal{B}$. Clearly $f=\widehat{\pi}(\widehat{g}) \in \mathcal{E}$.

To see (b), let $x \in X \backslash \sigma$ and $w \in \partial W(x)$. Note that $P-Q R \not \equiv 0$ since $\delta\left(x_{0}\right)=W\left(x_{0}\right)$ is nondegenerate. By Theorem 2, there is a unique $f_{0} \in \mathcal{E}$ such that $f_{0}(x)=w$ and, by Theorem 3 , we have $f_{0}\left(x_{0}\right) \in \partial W\left(x_{0}\right)=$ $\partial \delta\left(x_{0}\right)$. Therefore, there exists a unique $\zeta_{0} \in \partial D$ such that

$$
f_{0}\left(x_{0}\right)=\frac{P\left(x_{0}\right) \zeta_{0}+Q\left(x_{0}\right)}{R\left(x_{0}\right) \zeta_{0}+1} .
$$

The function $f=\left(P \zeta_{0}+Q\right) /\left(R \zeta_{0}+1\right)$ belongs to $\mathcal{E}$ by (a). As $f\left(x_{0}\right)=$ $f_{0}\left(x_{0}\right)$, Theorem 2 yields $f=f_{0}$ and $w=f_{0}(x)=f(x) \in \delta(x)$. This implies $\delta(x)=W(x)$. For $x \in \sigma, W(x)$ and hence $\delta(x)$ consist of the same single point.

We proceed to present a sufficient condition for parametrization.

TheOREM 4. If we have $\delta(x) \subset W(x)$ for any $x \in X \backslash \sigma$ and $\delta\left(x_{0}\right)=$ $W\left(x_{0}\right)$ for at least one $x_{0} \in X \backslash \sigma$, then the quadruple $(P, Q, R, 1)$ represents a Nevanlinna parametrization of $\mathcal{E}$.

Proof. Note that we see $P-Q R \not \equiv 0$ as in (b) of Lemma. For each $g \in \mathcal{B}$, we have $(P g+Q) /(R g+1) \in \mathcal{E}$ by (a) of Lemma. Conversely, let $f \in \mathcal{E}$. By (b) of Lemma, $\delta(x)=W(x)$ for all $x \in X$. Hence, for any $x \in X \backslash \sigma$, the linear mapping $\pi_{x}: \bar{D} \longrightarrow W(x)$ defined by

$$
\pi_{x}(\zeta)=\frac{P(x) \zeta+Q(x)}{R(x) \zeta+1} \quad(\zeta \in \bar{D})
$$

is bijective and we have $P(x)-Q(x) R(x) \neq 0$. The function

$$
g(x)=\pi_{x}^{-1}(f(x))=\frac{f(x)-Q(x)}{-R(x) f(x)+P(x)}
$$

is then holomorphic in $X \backslash \sigma$ and satisfies $|g| \leq 1$ there. The function $g$ may be regarded as a function in $\mathcal{B}$. Clearly $f=(P g+Q) /(R g+1)$. This shows 
that $(P, Q, R, 1)$ represents a Nevanlinna parametrization $\pi: \mathcal{B} \longrightarrow \mathcal{E}$ with its inverse $\pi^{-1}: \mathcal{E} \longrightarrow \mathcal{B}$ given by

$$
\pi^{-1}(f)=\frac{f-Q}{-R f+P} \quad(f \in \mathcal{E}) .
$$

Here, we would like to observe that there are many quadruples $(P, Q, R, 1)$ satisfying the condition $\delta(x) \subset W(x)$ for any $x \in X$ but representing no Nevanlinna parametrization of $\mathcal{E}$. Let $\left(P_{0}, Q_{0}, R_{0}, 1\right)$ be a quadruple representing a Nevanlinna parametrization of $\mathcal{E}$ and let $h \in \mathcal{B}$. Set $(P, Q, R, 1)=\left(P_{0} h, Q_{0}, R_{0} h, 1\right)$. For these functions $P, Q$, and $R$ holomorphic on $X$ and for $x \in X$, consider the set

$$
\begin{aligned}
\delta(x) & =\left\{\frac{P(x) \zeta+Q(x)}{R(x) \zeta+1}: \zeta \in \bar{D}\right\} \\
& =\left\{\frac{P_{0}(x) h(x) \zeta+Q_{0}(x)}{R_{0}(x) h(x) \zeta+1}: \zeta \in \bar{D}\right\} .
\end{aligned}
$$

Taking account of $|R(x) \zeta|<1$ for $\zeta \in \bar{D}$, we see at once that $\delta(x) \subset$ $W(x)$ for any $x \in X$ and that, for any $x \in X \backslash \sigma, \delta(x)=W(x)$ if and only if $|h(x)|=1$. By $h \in \mathcal{B}$ and by Theorem $4,(P, Q, R, 1)$ represents a Nevanlinna parametrization of $\mathcal{E}$ if and only if $h$ is a constant with $|h|=1$. This affirms the required assertion, since there are many $h \in \mathcal{B}$ such that $|h|<1$ on $X$. Finally, note that these functions $P, Q$, and $R$ with $S \equiv 1$ satisfy evidently the properties (a), (b), (c) and (d) indicated in the previous $\S 3$ if $h$ has no zeros on $X$. Thus, these properties give no sufficient condition for Nevanlinna parametrization.

\section{$\S 5$. An extension of Heins theorem}

In this section, let us extend Heins theorem to our extended interpolation problem.

Let $(\mathcal{I}, \mathfrak{c})$, where $\mathcal{I}=\left(\mathcal{I}_{x}\right)_{x \in X}$ and $\mathfrak{c}=\left(\mathfrak{c}_{x}\right)_{x \in X}$, be an extended interpolation problem on a simply connected Riemann surface $X$ of hyperbolic type. Let $\mathcal{B}, \mathcal{E}$, and $\sigma$ be as in the preceding section. We assume always $\sigma \neq \emptyset$. On the other hand, consider an analytic automorphism $T$ of $X$ and a Möbius transformation $U$ :

$$
U(w)=\lambda \frac{w+a}{1+\bar{a} w} \quad(\lambda, a \in \mathbb{C} ;|\lambda|=1,|a|<1) .
$$


Our present problem is to find a solution $f \in \mathcal{B}$ satisfying the interpolation condition

$$
f_{x}+\mathcal{I}_{x}=\mathfrak{c}_{x} \quad(\forall x \in X)
$$

and moreover the condition

$$
f \circ T=U \circ f
$$

For this purpose, it would be normal to impose on $(\mathcal{I}, \mathfrak{c})$ the following consistency conditions (5.1) and (5.2) and moreover the hypothesis of existence $\mathcal{E} \neq \emptyset$ :

$$
\begin{array}{cr}
T_{x}^{*}\left(\mathcal{I}_{T(x)}\right)=\mathcal{I}_{x} & (\forall x \in X) ; \\
T_{x}^{*}\left(\mathfrak{c}_{T(x)}\right)=U_{x}\left(\mathfrak{c}_{x}\right) & (\forall x \in \sigma) .
\end{array}
$$

Note that, under the hypothesis $\mathcal{E} \neq \emptyset, U_{x}\left(\mathfrak{c}_{x}\right)$ is defined for each $x \in \sigma$, as was pointed out in $\S 2$, because we have $\{0\} \neq \mathcal{I}_{x} \neq \mathcal{O}_{x}$ and $\mathfrak{c}_{x}=f_{x}+\mathcal{I}_{x}$ for some $f \in \mathcal{B}$, which implies that we have $1+\bar{a} f(x) \neq 0$ and hence $1+\bar{a} \mathfrak{c}_{x}$ is a unit of $\mathcal{O}_{x} / \mathcal{I}_{x}$.

We are now ready to establish

TheOREm 5. Suppose $\mathcal{E} \neq \emptyset$ and that the consistency conditions (5.1) and (5.2) are fulfilled. Then, there exists a function $f \in \mathcal{E}$ satisfying

$$
f \circ T=U \circ f .
$$

Proof. Let $f \in \mathcal{E}$. We claim that $U^{-1} \circ f \circ T \in \mathcal{E}$ and $U \circ f \circ T^{-1} \in \mathcal{E}$. Clearly, $U^{-1} \circ f \circ T \in \mathcal{B}$ and $U \circ f \circ T^{-1} \in \mathcal{B}$. For any $x \in \sigma$, we have $\left(U^{-1} \circ f \circ T\right)_{x}+\mathcal{I}_{x}=\left(U^{-1}\right)_{x}\left((f \circ T)_{x}+\mathcal{I}_{x}\right)=U_{x}^{-1}\left(T_{x}^{*}\left(f_{T(x)}+\mathcal{I}_{T(x)}\right)\right)=$ $U_{x}^{-1}\left(T_{x}^{*}\left(\mathfrak{c}_{T(x)}\right)\right)=\mathfrak{c}_{x}$ and $\left(U \circ f \circ T^{-1}\right)_{T(x)}+\mathcal{I}_{T(x)}=\left(T^{-1}\right)_{T(x)}^{*}\left((U \circ f)_{x}+\right.$ $\left.\mathcal{I}_{x}\right)=\left(T_{x}^{*}\right)^{-1}\left(U_{x}\left(\mathfrak{c}_{x}\right)\right)=\mathfrak{c}_{T(x)}$, by definition and by (5.1) and (5.2). For any $x \in X \backslash \sigma$, we have to say nothing.

In the case where $\mathcal{E}$ has only one element $f$, we have $f=U^{-1} \circ f \circ T$, which proves the theorem.

Now, assume $\mathcal{E}$ has at least two elements. Then, as was mentioned in $\S 3$, we may take a Nevanlinna parametrization $\pi$ of $\mathcal{E}$ represented by a quadruple $(P, Q, R, S)$. Consider for each $x \in X$ the set $W(x)=\{f(x)$ : $f \in \mathcal{E}\}$. We claim that

$$
W(x)=U^{-1}(W(T(x))) \quad(\forall x \in X) .
$$


In fact, if $f \in \mathcal{E}$ and $x \in X$, then $f(T(x))=U\left(\left(U^{-1} \circ f \circ T\right)(x)\right) \in U(W(x))$ and $U(f(x))=\left(U \circ f \circ T^{-1}\right)(T(x)) \in W(T(x))$. This shows $W(T(x))=$ $U(W(x))$ and hence (5.4) holds.

Keeping in mind the expression

$$
U^{-1}(w)=\frac{w-\lambda a}{\lambda-\bar{a} w}
$$

and defining four holomorphic functions $P_{0}, Q_{0}, R_{0}$, and $S_{0}$ on $X$ by

$$
\left[\begin{array}{ll}
P_{0} & Q_{0} \\
R_{0} & S_{0}
\end{array}\right]=\left[\begin{array}{cc}
1 & -\lambda a \\
-\bar{a} & \lambda
\end{array}\right]\left[\begin{array}{ll}
P & Q \\
R & S
\end{array}\right]
$$

we can write by (5.4)

$$
\begin{aligned}
W(x) & =U^{-1}\left(\left\{\frac{P(T(x)) \zeta+Q(T(x))}{R(T(x)) \zeta+S(T(x))}: \zeta \in \bar{D}\right\}\right) \\
& =\left\{\frac{P_{0}(T(x)) \zeta+Q_{0}(T(x))}{R_{0}(T(x)) \zeta+S_{0}(T(x))}: \zeta \in \bar{D}\right\},
\end{aligned}
$$

which is in $D$.

By Proposition in $\S 4$, the functions

$$
\widehat{P}(x)=\frac{P_{0}(T(x))}{S_{0}(T(x))}, \quad \widehat{Q}(x)=\frac{Q_{0}(T(x))}{S_{0}(T(x))}, \quad \widehat{R}(x)=\frac{R_{0}(T(x))}{S_{0}(T(x))}
$$

are holomorphic and verify $|\widehat{R}|<1$, first in $X \backslash \sigma$ and hence on $X$ entirely. From

$$
W(x)=\left\{\frac{\widehat{P}(x) \zeta+\widehat{Q}(x)}{\widehat{R}(x) \zeta+1}: \zeta \in \bar{D}\right\} \quad(\forall x \in X)
$$

and from Theorem 4 in $\S 4$, it follows that $(\widehat{P}, \widehat{Q}, \widehat{R}, 1)$ represents a Nevanlinna parametrization $\widehat{\pi}$ of $\mathcal{E}$. By Theorem 1, we find a unique Möbius transformation $\widehat{U}$ such that

$$
\pi(g)=\widehat{\pi}(\widehat{U} \circ g) \quad(\forall g \in \mathcal{B}) .
$$

Let us see that, for $g \in \mathcal{B}, f=\pi(g)$ satisfies $f \circ T=U \circ f$ if and only if $g$ satisfies the condition

$$
g \circ T=\widehat{U} \circ g
$$


In fact, for $x \in X$, we have by definition

$$
\begin{aligned}
\left(U^{-1} \circ \pi(g) \circ T\right)(x) & =U^{-1}\left(\frac{P(T(x)) g(T(x))+Q(T(x))}{R(T(x)) g(T(x))+S(T(x))}\right) \\
= & \frac{\widehat{P}(x) g(T(x))+\widehat{Q}(x)}{\widehat{R}(x) g(T(x))+1}=(\widehat{\pi}(g \circ T))(x)=\pi\left(\widehat{U}^{-1} \circ g \circ T\right)(x) .
\end{aligned}
$$

Therefore $f=\pi(g)=U^{-1} \circ f \circ T$ if and only if $g=\widehat{U}^{-1} \circ g \circ T$, which shows the assertion.

The theory of linear transformations permits us to take a fixed point $\zeta$ of $\widehat{U}$ in $\bar{D}$. When $g$ is the constant $\zeta, g$ satisfies evidently (5.5) and hence $f=\pi(g) \in \mathcal{E}$ satisfies the condition (5.3). The theorem is established.

We wish to conclude this paper with applying Theorem 5 to the extended interpolation problem on the annuli, just as Heins did in [5] for the Pick-Nevanlinna interpolation problem.

Let $X$ be a doubly connected Riemann surface biholomorphically equivalent to an annulus $\{z \in \mathbb{C}: 1<|z|<\rho\}(1<\rho<\infty)$ and let $(\mathcal{I}, \mathfrak{c})$, where $\mathcal{I}=\left(\mathcal{I}_{x}\right)_{x \in X}$ and $\mathfrak{c}=\left(\mathfrak{c}_{x}\right)_{x \in X}$, be an extended interpolation problem on $X$. Choose a holomorphic mapping $\varphi: D \longrightarrow X$ of the open unit disc $D$ onto $X$ such that $(D, \varphi)$ is a universal covering of $X$. For each $z \in D$, setting

$$
\widetilde{\mathcal{I}}_{z}=\varphi_{z}^{*}\left(\mathcal{I}_{\varphi(z)}\right), \quad \widetilde{\mathcal{I}}=\left(\widetilde{\mathcal{I}}_{z}\right)_{z \in D} \quad \text { and } \quad \tilde{\mathfrak{c}}_{z}=\varphi_{z}^{*}\left(\mathfrak{c}_{\varphi(z)}\right), \quad \widetilde{\mathfrak{c}}=\left(\widetilde{\mathfrak{c}}_{z}\right)_{z \in D}
$$

we have an extended interpolation problem $(\widetilde{\mathcal{I}}, \widetilde{\mathfrak{c}})$ on $D$.

Corollary. A necessary and sufficient condition that there exists a holomorphic function $f$ on $X$ satisfying

$$
|f| \leq 1 \quad \text { on } X \quad \text { and } \quad f_{x}+\mathcal{I}_{x}=\mathfrak{c}_{x} \quad(\forall x \in X)
$$

is that there exists a holomorphic function $\tilde{f}$ on $D$ satisfying

$$
|\tilde{f}| \leq 1 \quad \text { on } D \quad \text { and } \quad \tilde{f}_{z}+\tilde{\mathcal{I}}_{z}=\widetilde{\mathfrak{c}}_{z} \quad(\forall z \in D)
$$

In fact, if $f$ is such a function on $X$, then it is not hard to see that $\widetilde{f}=f \circ \varphi$ satisfies the second conditions. Conversely, let $\widetilde{f}$ be such a function on $D$. In Theorem 5 , let $U: \bar{D} \longrightarrow \bar{D}$ be the identity, replace $X$ by $D$, and let $T: D \longrightarrow D$ be a generator of the covering transformation group 
of $(D, \varphi)$. We have $T_{z}^{*}\left(\widetilde{\mathcal{I}}_{T(z)}\right)=T_{z}^{*}\left(\varphi_{T(z)}^{*}\right)\left(\mathcal{I}_{\varphi(T(z))}\right)=(\varphi \circ T)_{z}^{*}\left(\mathcal{I}_{(\varphi \circ T)(z)}\right)=$ $\varphi_{z}^{*}\left(\mathcal{I}_{\varphi(z)}\right)=\widetilde{\mathcal{I}}_{z}$ and $T_{z}^{*}\left(\widetilde{\mathfrak{c}}_{T(z)}\right)=T_{z}^{*}\left(\varphi_{T(z)}^{*}\right)\left(\mathfrak{c}_{\varphi(T(z))}\right)=\varphi_{z}^{*}\left(\mathfrak{c}_{\varphi(z)}\right)=\widetilde{\mathfrak{c}}_{z}$. As the consistency conditions are thus verified, we may assume $\widetilde{f} \circ T=\widetilde{f}$ by Theorem 5. Then there exists a holomorphic function $f$ on $X$ such that $f \circ \varphi=\tilde{f}$. Clearly $|f| \leq 1$ on $X$. As $\varphi_{z}$ is isomorphic for any $z \in D$, we see at once $f_{x}+\mathcal{I}_{x}=\mathfrak{c}_{x}(\forall x \in X)$.

\section{REFERENCES}

[1] M. B. Abrahamse, The Pick interpolation theorem for finitely connected domains, Michigan Math. J., 26 (1979), 195-203.

[2] L. V. Ahlfors, Conformal Invariants. Topics in Geometric Function Theory, McGraw-Hill, New York, 1973.

[3] S. D. Fisher, Function Theory on Planer Domains, Wiley, New York, 1983.

[4] J. B. Garnett, Bounded Analytic Functions, Academic Press, New York, 1981.

[5] M. H. Heins, Extremal problems for functions analytic and single-valued in a doubly-connected region, Amer. J. Math., 62 (1940), 91-106.

[6] — Nonpersistence of the grenzkreis phenomenon for Pick-Nevanlinna interpolation on annuli, Ann. Acad. Sci. Fenn. A. I., 596 (1975).

[7] D. E. Marshall, An elementary proof of Pick-Nevanlinna interpolation theorem, Michigan Math. J., 21 (1974), 219-223.

[8] R. Nevanlinna, Über beschränkte Funktionen die in gegebenen Punkten vorgeschriebene Werte annehmen, Ann. Acad. Sci. Fenn., 13, No 1 (1919).

[9] — Über beschränkte analytische Funktionen, Ann. Acad. Sci. Fenn. Ser A, 32, No 7 (1929).

[10] G. Pick, Über die Beschränkungen analytischer Funktionen, welche durch vorgegebene Funktionswerte bewirkt werden, Math. Ann., 77 (1916), 7-23.

[11] I. Schur, Über Potenzreihen, die im Innern des Einheitskreises beschränkt sind, J. Reine Angew. Math., 147 (1917), 205-232.

[12] A. Stray, Minimal interpolation by Blaschke products II, Bull. London Math. Soc., 20 (1988), 329-332.

[13] S. Takahashi, Extension of the theorems of Carathéodory-Toeplitz-Schur and Pick, Pacific J. Math., 138 (1989), 391-399.

[14] Levanlinna parametrizations for the extended interpolation problem, Pacific J. Math., 146 (1990), 115-129.

[15] — Extended interpolation problem in finitely connected domains, Oper. Theory Adv. Appl., 59 (1992), 305-327. 
Department of Mathematics

Nara Women's University

Nara 630-8506

Japan

sechiko@cc.nara-wu.ac.jp 